\title{
Stokesmeter observations of large-scale solar magnetic fields in different spectral lines, and diagnostics of fine-structure magnetic elements
}

\author{
Michael L. Demidov and Renat M. Veretsky
}

Department of Solar Physics, Institute of Solar-Terrestrial Physics at the Siberian Branch of the Russian Academy of Sciences, Irkutsk, P.O.Box 4026, Russia. E-mail: demid@iszf.irk.ru

\begin{abstract}
The temperature, velocity and magnetic field structures of solar atmosphere are extremely in-homogeneous under consideration on a small spatial scales. This fact is the reason of significant difficulties in the attempts of disambiguous interpretation of observations. Particularly informative for investigation of the complex polarized radiation formation processes in the dynamic solar atmosphere with a fine-structured organization of magnetic fields are the distributions across the solar disk of the amplitude asymmetry $(\delta a)$ and area asymmetry $(\delta A)$ of Stokes profiles, as well as of the strength ratios $R$ in different combinations of spectral lines. At this paper such information has been obtained using the Stokesmeter of the STOP telescope at the Sayan observatory. It was found that for some Fraunhofer lines the distribution of $R$ has an evident equatorial-polar asymmetry, which is difficult to explain yet. Additionally this paper presents some new results of the theoretical simulation (with different parameters of magnetic flux tubes models and of the medium in their neighborhood) of the center-to-limb variations of $\delta A$ on the example of the FeI $\lambda 525.02 \mathrm{~nm}$ spectral line and of the $R=B_{525.02} / B_{524.70}$. The conclusion is made that a reasonably good agreement theory and experimental data can be achieved, for the time being, only for the central regions of the disk.
\end{abstract}

\section{Introduction}

An investigations of the Sun with as high spatial resolution as possible, including the observations of magnetic fields are very important from different points of view. Some interesting reports presented at this symposium perfectly prove this statement. Nevertheless, observations with moderate and even with low spatial resolution did not lose their topicality either. It is possible to remind an observations of the mean magnetic field of the Sun-as-a-star and observations of distribution of the large-scale magnetic fields (LSMF) across the solar disk. Such kind observations are still very important for studies of the global properties and evolution of solar magnetism. Recently, a new additional aspect has appeared connected with using these observations for calculations of magnetic fields in the corona and in the interplanetary medium in the frames of space weather program. But up to now, the situation with reliable quantitative interpretation of solar magnetic field measurements is far from understanding. The reasons are the very complicated fine-structured organization of temperature, velocity and magnetic fields in the solar atmosphere. This makes the theoretical description of the polarized radiation transfer by the extremely difficult problem.

There is a hope that a significant progress in a solution of at least some of these problems can be achieved with involving a new spectropolarimetric observations that have become available with development of the Stokesmeter on the STOP telescope at the Sayan observatory [Demidov et al (2002)]. This instrument allows to obtain a high-precision Stokesmeter LSMF measurements (typically with angular resolution about 

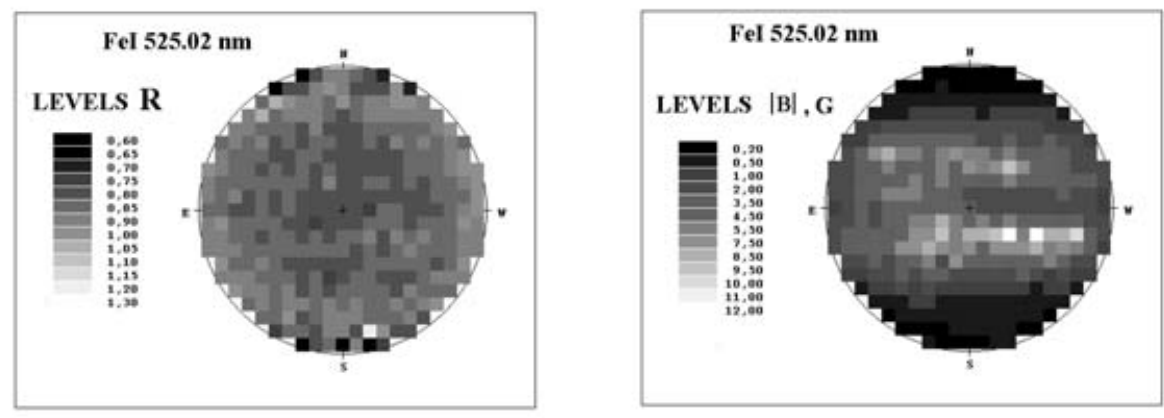

Figure 1. The distribution across the solar disk of the $R=B_{525.02} / B_{524.70}$ (left panel) and of the absolute magnetic field strengths (right panel).

100") across the whole solar disk simultaneously in several spectral lines. Previously a Stokesmeter observations were available as a rule only in the specially selected solar surface areas. Earlier with usage a qualitatively new observing material an interesting results concerning the distribution across the solar disk of the amplitude $(\delta a)$ and area $(\delta A)$ asymmetries of the Stokes $\mathrm{V}$-profiles have been obtained on the example of the FeI 525.02 nm spectral line [Demidov et al (2001)]. As it well known, these parameters are extremely informative for study of the complex dynamical processes in the magnetic flux tubes (MFT) and their neighborhoods. In the paper [Demidov \& Veretsky (2003)] it was shown that the experimental center-to-limb variation (CLV) of the $\delta a$ can be rather good reproduced by the theoretical calculations. At present paper the main attention is concentrated on the new results concerning the spatial distributions across the solar disk of the magnetic strength ratios $R$ in different combination of spectral lines in the vicinity of the FeI $\lambda 525.02 \mathrm{~nm}$. Quite unexpectedly a significant asymmetry in CLV of the $R$ in the polar and equatorial directions for some pairs of line was discovered. Besides, the aim of this paper is to present some new theoretical simulation results of the $R$ and $\delta A$ CLV.

\section{Observations}

The most known spectral lines widely used for diagnostics of the internal invisible structure of the solar magnetic fields are the FeI $\lambda 524.70 \mathrm{~nm}$ and FeI $\lambda 525.02 \mathrm{~nm}$. At some previous papers the CLV of the $R=B_{525.02} / B_{524.70}$ has been studied from experimental and theoretical points of view (see Demidov et al (2003) and references there). But it is certainly very interesting to know a two-dimensional distribution of this parameter across the solar disk. With using of 35 observation days such distribution has been obtained and presented here in Fig.1 (left panel). For the comparison on the right panel of Fig.1 the distribution of the absolute values of the magnetic field strengths (magnetic fluxes) is presented. It is possible to see that there is no evident connection between these two maps. As concern of the $R$ map a remarkable differences between the near-limb values at the pole and equator areas might be noticed.

Of course, it is important to exame: does this phenomena of polar-equatorial $R$ asymmetry valid only for the FeI $\lambda 524.70 \mathrm{~nm}$ and FeI $\lambda 525.02 \mathrm{~nm}$, or for the other spectral lines combinations as well? To answer this questions the two-dimensional maps of $R$ parameter for all combinations of four lines in the vicinity of the FeI $\lambda 524.70 \mathrm{~nm}$ have been constructed. Then on the basis of these maps the CLV of $R$ for combined polar (NS) and equatorial (WE) sectors have been calculated, The results are presented in Fig.2. 

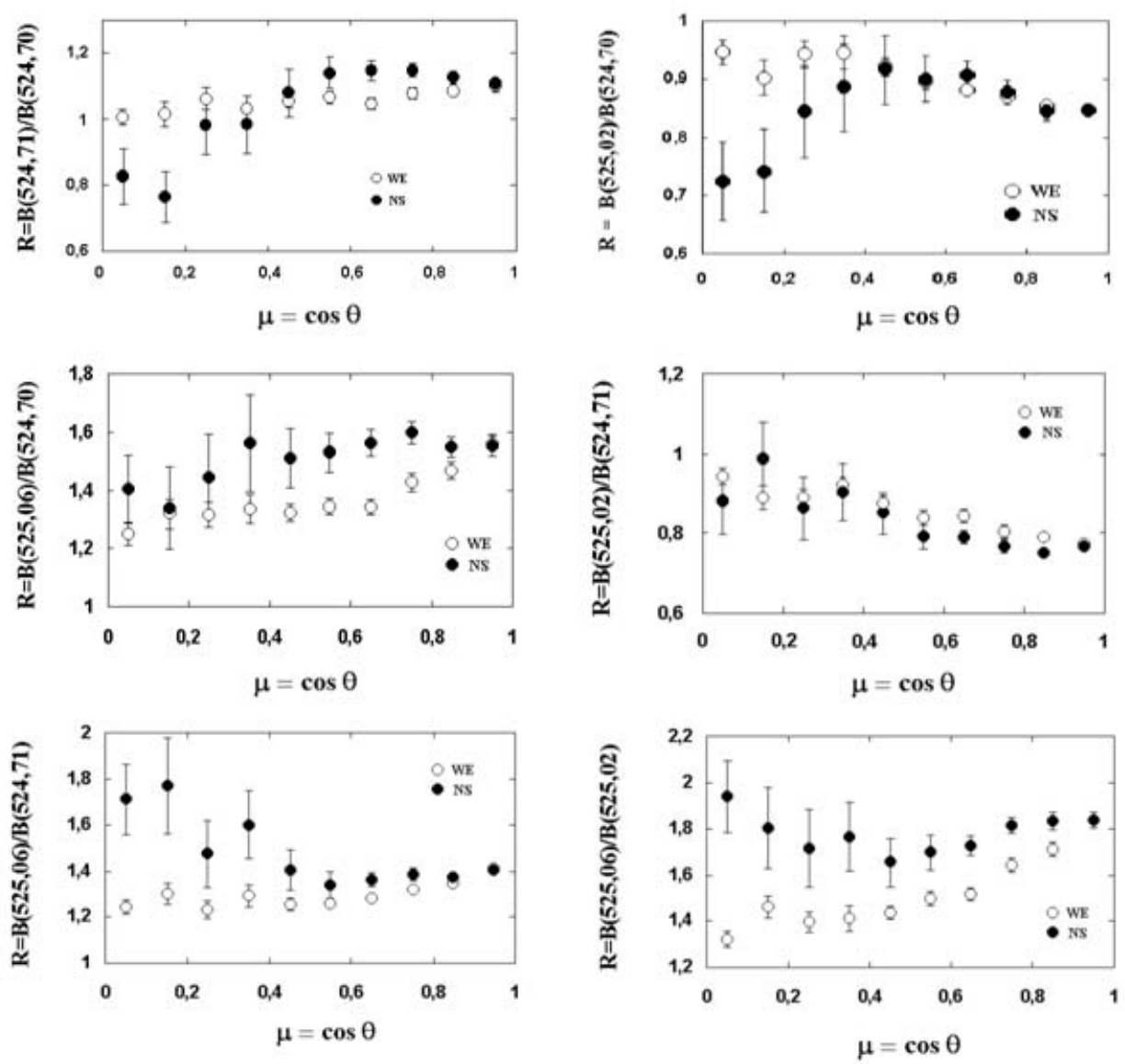

Figure 2. The center-to-limb-variations of magnetic strength ratios $R$ in different combinations of spectral lines for polar (NS) and equatorial (EW) sectors

Obviously, the differences between NS and WE curves depends on the combinations of spectral lines. For an example, in case of CrI $\lambda 524.71 \mathrm{~nm}-$ FeI $\lambda 525.02 \mathrm{~nm}$ and FeI $\lambda 524.70 \mathrm{~nm}-\mathrm{FeI} \lambda 525.06 \mathrm{~nm}$ the differences are small. Otherwise, for combination FeI $\lambda 525.02 \mathrm{~nm}-$ FeI $\lambda 525.06 \mathrm{~nm}$ there is a significant difference starting already since the small distances from the disk center.

\section{Theoretical simulation of the experimental data}

At present time there is no clear idea yet how to explain the discovered existence of the polar-equatorial asymmetry in the CLV of $R$ in some combinations of spectral lines. Most probably, the reason for this phenomena could be the influence of solar rotation and/or of the toroidal component of magnetic fields. An additional careful calculations with different assumptions are necessary.

At present time the attempts to theoretically reproduce only an average CLV (i.e. 

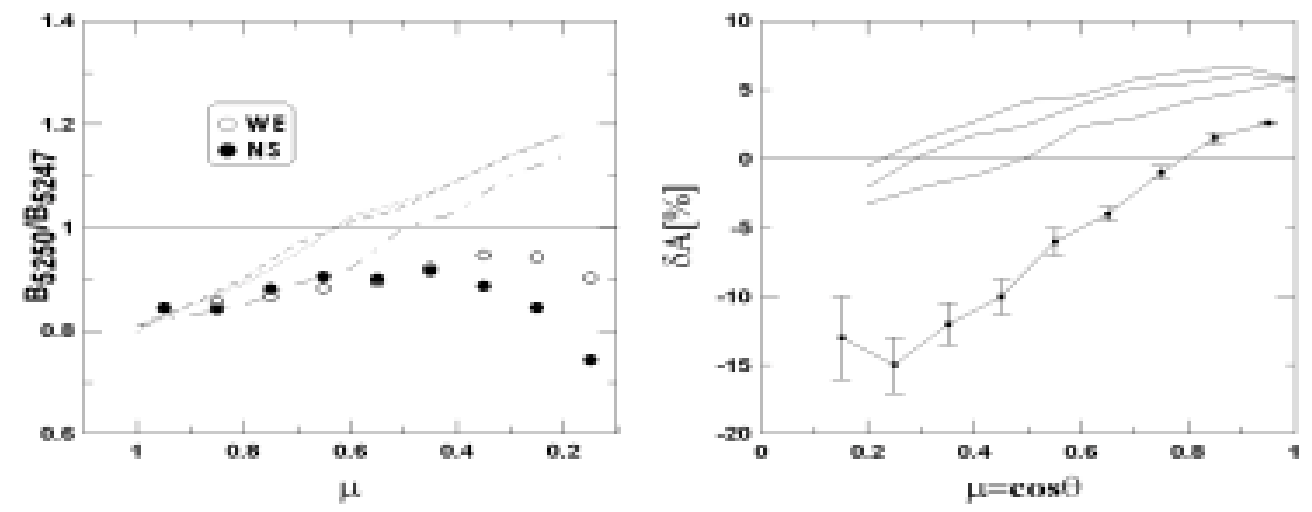

Figure 3. Comparison of the experimental and theoretical CLV of the $R=B_{525.02} / B_{524.70}$ (left panel) and of the $\delta A$ for the line FeI $\lambda 525.02 \mathrm{~nm}$ (right panel). The details see in the text.

without separation on the sectors) and only of the parameters $R=B_{525.02} / B_{524.70}$ and $\delta A$ for the line FeI $\lambda 525.02 \mathrm{~nm}$ have been made. It was supposed that MFT are embedded in the field-free plasma with the determined velocity structure (different for calculations of the $R$ and $\delta A$ ). The results of calculations with different assumptions in comparison with experimental data are shown in Fig.3. On the left panel the calculations for three types of the MFT arrays with different radius $r$ of the tubes and of the filling factor values $f$ at the zero level are presented: (1) $r=100 \mathrm{~km}, \mathrm{f}=5 \%$ (dashed line); $2 . r=100$ $\mathrm{km}, \mathrm{f}=10 \%$ (dotted line); 3. $r=200 \mathrm{~km}, \mathrm{f}=5 \%$ (dot-dashed line). The more-detailed information about the procedure of calculations and accepted restrictions can be found in [Demidov \& Veretsky (2003)]. Quite different assumptions (similar to the offered ones in the paper [Bünte et al (1993)]concerning the velocity fields between MFT have been used for CLV simulation of the $\delta A$. The results of calculations for the first type of the MFT array for three maximum radial velocity values (the upper theoretical curve corresponds to $2.5 \mathrm{~km} / \mathrm{s}$, the low curve - to $5 \mathrm{~km} / \mathrm{s}$, and the intermediate one - to $4 \mathrm{~km} / \mathrm{s}$ ) are plotted on the right panel. From the consideration of Fig. 3 the conclusion can be made that a considerable difference between the experimental and numerical results takes place on the big heliocentric distances from the solar disk center and that this discrepancy awaits its explanation in future studies.

\section{Acknowledgements}

We are grateful to Drs. V.M. Grigoryev, V.S. Peshcherov and T.A. Latushko for the discussions, participation in the observations and data processing.

The results presented in this paper were obtained though partial support by the INTAS grant 2000-840 and RFBR grant 02-02-16467.

\section{References}

Bünte, M., Solanki, S. K., \& Steiner, O. 1993 A \& A 268, 736-748.

Demidov, M.L. \& Veretsky R.M. 2003 ASP Conference Series 286, 133-138.

Demidov, M.L., Veretsky R.M. \& Peshcherov, V.S. 2003 ASP Conference Series 307, 352-357.

Demidov, M.L., Peshcherov, V.S. Zhigalov, V.V. \& Grigoryev, V.M. 2001 ASP Conference Series 236, 471-478.

Demidov, M.L., Zhigalov, V.V., Peshcherov, V.S. \& Grigoryev, V.M. 2002 Solar Phys. 209, 217-232. 